International Journal of Pure and Applied Mathematics

Volume $91 \quad$ No. 4 2014, 483-493

ISSN: 1311-8080 (printed version); ISSN: 1314-3395 (on-line version)

url: http://www.ijpam.eu

doi: http://dx.doi.org/10.12732/ijpam.v91i4.5

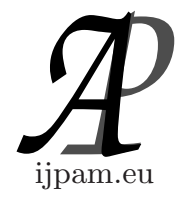

\title{
THE PRINCIPLE OF MACH IN NEWTONIAN CONTINUUM THERMOMECHANICS
}

\author{
Trevor H. Moulden \\ Department of Mechanical and Aerospace Engineering \\ The University of Tennessee Space Institute \\ Tullahoma, Tennessee, 37388, USA
}

\begin{abstract}
The paper develops a Newtonian version of Mach's principle for continuum thermomechanics. The starting point is the covariant formulation of Newtonian thermomechanics for the entire (Newtonian) universe.
\end{abstract}

AMS Subject Classification: 74Axx, 76Axx

Key Words: continuum mechanics, mach principle

\section{Background}

The Galilei-Newton principle of inertia was expressed by Newton in the form (see Newton [11] as given in the translation by Cohen and Whitman [4]):

Axiom N1. Axiom of inertia

"Every body preserves in its state of being at rest or of moving uniformly straight forward except insofar as it is compelled to change its state by forces impressed".

for the mechanics of rigid bodies and point masses. Axiom N1 serves to establish the background spacetime for Newtonian mechanics and the transformation group acting on that spacetime. The resulting inertial frames, from equation (1) below, are realized, to very good approximation, in many situations of practical interest. Newton [11] suggested, in Definition 3, of the "Principia"

Received: December 4, 2013

(c) 2014 Academic Publications, Ltd. url: www.acadpubl.eu 
that bodies had the property such that the "inherent force of matter is the power of resisting by which every body, so far as it is able, preserves in its state either of resting or of moving uniformly straight forward". "Resting" and "moving" here are relative to Newton's absolute space. Such an inherent force could not be of the same nature as the forces specified in the second axiom of Newton so that the ontological structure, and the implications, of the first axiom remain rather opaque. The principle of Mach was put forward as an attempt to provide a consistent structure for Newtonian mechanics. To quote from Mach [7]: "... the neglect of the rest of the world is impossible." when discussing inertial properties of bodies. As shown below, this principle of Mach does, in the Newtonian context, provide an expression for inertia in terms of an integral over the external universe. But, again, no mechanism for the inertial property is provided by this global formulation.

Mach [7], [8] was not the first to call attention to problems with the formulation of the mechanics as given by Newton [11]. In particular, Berkeley wrote that motion could not be detected in a one-body universe contrary to Newton's requirements in the construction of absolute space. Berkeley [2] (see Section \#112) noted that “... to conceive motion, there must be at least conceived two bodies, ...". Berkeley's one-body universe could be construed as the introduction of a proto-Machian concept. A more complete discussion of the background to the principle of Mach, along with a historical perspective, is contained in Barbour [1].

Axiom N1 was recently expanded to treat the case of Newtonian continuum thermomechanics (see Moulden [9]) and this extension, as well as the attendant notation, will be adopted herein without comment. The present interest resides in an understanding of the Mach principle in the context of Newtonian continuum thermomechanics and constitutes a restatement of Moulden [10]. That is, the present development adopts the Galilean spacetime of Newtonian mechanics rather than the spacetime associated with the mechanics of Mach (see Earman [5] for details of Machian spacetime).

It is well known that the axioms of Newtonian mechanics leads to the socalled Galilean subgroup, $\mathbb{G}_{a}$, of the group of Euclidean transformations on Newtonian spacetime $\mathbb{W}_{s t}$. Let $\Lambda_{c}$ be the canonical coordinate frame on $\mathbb{W}_{s t}$ with its origin at the mass center of the universe, 0 . Here $\mathbf{x} \in \mathbb{S}_{t}$ is a vector in the subspace of simultaneity, at time $t$, of $\mathbb{W}_{s t}$. $\mathbb{T}$ represents the corresponding time axis. The subgroup $\mathbb{G}_{a}$ will be defined in the standard form:

$$
\mathbf{x}^{*}=\mathbf{Q}_{G} \mathbf{x}+\mathbf{V}_{T} t+\mathbf{x}_{0} ; \quad t^{*}=t+t_{0}
$$

as required by axiom $\mathrm{N} 1$. Here $\mathbf{Q}_{G}$ represents a constant coordinate orientation 
change and $\mathbf{V}_{T} \in \mathbb{R}^{3}$ a bounded constant boost velocity of frame $\Lambda^{*}$ relative to frame $\Lambda_{c} . \mathbf{x}_{0}$ represents a possible (bounded) constant spatial translation of the coordinate origin.

The Newtonian group, $\mathbb{G}_{n}$, of transformations on $\mathbb{W}_{s t}$ is in two parts: the Galilean transformations, in equation (1) and the axial group, $\mathbb{G}_{z}$, defined by the equality:

$$
\mathbf{x}^{*}=\mathbf{Q}(t) \mathbf{x}
$$

where $\mathbf{Q}(t)=\exp [\mathbf{Z} t] \in S O_{3}$. The coordinate spin, $\mathbf{Z}=\mathbf{Q}^{T} \dot{\mathbf{Q}}$, being a constant skew symmetric operator. Let $\mathbf{r}$ identify the axial vector of $\mathbf{Z}$. The axial group defines the rotational inertia properties of bodies which Newton [11] described as: "A spinning hoop, $\cdots$, does not cease to rotate, except insofar as it is retarded by the air". Section 4 below will discuss the angular Mach principle.

Introduce the main result in the following form:

Theorem 1.1 The principle of Mach for Newtonian mechanics.

The theorem is in three distinct parts:

a). The Newtonian group is the appropriate group for this mechanics.

b). The linear inertia of body $\mathcal{B}$ can be expressed in terms of properties of the component, $B^{e}$, of the universe that is exterior to $\mathcal{B}$.

c). The angular inertia of body $\mathcal{B}$ : rotation, with constant angular velocity of $\mathcal{B}$ about a fixed axis through the mass center of that body, is a privileged motion.

Part (a) of the theorem is classical and was discussed in Moulden [9] and needs no further comment. The inertial properties contained in (b) and (c) can be understood from an application of the covariant formulation of continuum thermomechanics to the entire (Newtonian) universe. Again, these two aspects of inertial motion, angular and linear, are distinct and are the consequences of the different components associated with the Newtonian group of transformations on $\mathbb{W}_{s t}$. The background to theorem 1.1 leaves aside questions concerning the validity of Newtonian mechanics on a global scale.

Let $\mathcal{B}=\left\{\delta \mathcal{B}_{i}\right\}$ be a continuous body, with body points $\delta \mathcal{B}_{i}$, that is in bijective correspondence with a region $\mathcal{D}_{t} \subset \mathbb{R}^{3}$ of physical space at time $t \in R$.

Definition 1.1 The Newtonian Universe, 0 .

The collection $00=\left\{\mathcal{B}_{i}\right\}$ of bodies $\mathcal{B}$ in 00 define the Newtonian universe. This universe is contained in a fixed compact region of three-dimensional Euclidean space for all time. No agencies exterior to the boundary, 200 , of 00 influence the thermomechanics of its interior.

and represents the universe as understood at the time of Newton. The 
bodies are treated in terms of Newtonian continuum mechanics and may, or may not, be separated by the void, or empty body, $\mathcal{O}$. All bodies in the universe have a non-negative mass with only the void, $\mathcal{O}$, having zero mass. The notation:

Definition 1.2 Contiguous bodies

Body $\mathcal{B}_{c}=\mathcal{B}_{i} \curlyvee^{c} \mathcal{B}_{j}$ is said to be contiguous if $\mathcal{B}_{c}=\mathcal{B}_{i} \cup \mathcal{B}_{j}$ with int $\left(\mathcal{B}_{i}\right) \cap$ $\operatorname{int}\left(\mathcal{B}_{j}\right)=\emptyset$ but the intersection $\mathcal{B}_{i} \cap \mathcal{B}_{j}=\partial \mathcal{B}_{i j} \neq \emptyset$ for $\mathcal{B}_{i}$ and $\mathcal{B}_{j}$ in $\mathbb{B}(\mathcal{B})$. $\mathcal{B}_{i}$ and $\mathcal{B}_{j}$ are said to be the contiguous parts of $\mathcal{B}_{c}$ with $\partial \mathcal{B}_{i j}$ their common interface. Note that $\partial \mathcal{B}_{i j} \subset \partial \mathcal{B}_{i}$ and $\partial \mathcal{B}_{i j} \subset \partial \mathcal{B}_{j} . \mathcal{D}_{c} \subset \mathbb{S}_{t}$ is the space occupied by $\mathcal{B}_{c}$ at time $t$.

as is standard (and copied from Moulden [9]). Then $O O=\mathcal{O} \curlyvee_{i}^{c} \mathcal{B}_{i}$ is the contiguous body formed from the join of all physical bodies, $\mathcal{B}_{i}$, in the universe with the void, $\mathcal{O}$. Time is taken to be absolute, as in the Newtonian theory, with one time scale for the entire universe. The void is devoid of all physical properties since Newtonian mechanics ignores any quantum mechanical structure that may exist in the vacuum of the real universe. It is necessary to make:

Axiom 1.1 The Universe; its body and mass.

The universe 00 is finite and isolated with no preferred structure or orientation. It occupies a bounded subset of the space $\mathbb{S t}$ for all time. In addition, the mass $M(0)>0$, is time invariant as well as finite.

This time invariance of mass holds for each component body, $\mathcal{B}_{i}$, in the universe with each $M\left(\mathcal{B}_{i}\right) \geq 0$ time invariant. It is assumed that the universe contains a countable number of bodies. A comment can be made:

Requirement 1.1 The material derivative, $d(\cdot) / d t$, must commute with the body mass integral $M(\mathcal{B})=\int_{\mathcal{B}}(\cdot) d m . \quad \square$ since an integral over $\mathcal{B}$ with respect to mass is independent of body location. Here $d m$ is the local mass measure over the body $\mathcal{B}$. Then the obvious result follows:

Lemma 1.1 With the conservation principle $d M(\mathcal{B}) / d t \equiv 0$ in place, $d / d t$ commutes with $\int_{\mathcal{B}}(\cdot) d m$ iff:

$$
d \rho / d t+\rho \operatorname{div}(\mathbf{v})=0
$$

Proof. From the definition, the transport and the localization theorems.

Here, $\rho(\mathbf{x}, t) \geq 0$ is the local mass density of the body. 


\section{Covariant Structure}

The required background to the covariant structure of Newtonian continuum thermomechanics was given in Moulden [9] and based upon the statement of Clausius which demanded that the total energy of the universe be time invariant. Thus, write:

Definition 2.1 The total energy $\mathrm{E}_{T}(\mathcal{B})$

Let $\mathcal{B}$ be a thermomechanical body then:

$$
\mathrm{E}_{T}(\mathcal{B})=\int_{\mathcal{B}}[e(\mathbf{x}, t)+\langle\mathbf{v}, \mathbf{v}\rangle / 2] d m
$$

is the total energy of body $\mathcal{B}$. Here $e(\mathbf{x}, t)$ is the specific internal energy associated with $\mathcal{B}$ as a continuous field over $\mathcal{B}$ and $\mathbf{v}(\mathbf{x}, t)$ denotes the local velocity vector of each body point.

Next, introduce the quantity, the so-called action, $\mathrm{A}(\mathcal{B} ; t)$, from Moulden [9], where:

Definition 2.2 The action $\mathrm{A}(\mathcal{B} ; t)$.

Introduce:

$$
\mathrm{A}(\mathcal{B} ; t) \equiv \frac{d}{d t} \mathrm{E}_{T}(\mathcal{B})=\int_{\mathcal{B}}\left[\frac{d e}{d t}+\langle\mathbf{a}, \mathbf{v}\rangle\right] d m
$$

as an integral over the body $\mathcal{B}$, with $\mathbf{a}(\mathbf{x}, t)$ the local acceleration field.

The full energy equation is identified from the equality:

$$
\mathrm{A}(\mathcal{B} ; t)=\mathrm{P}\left(\mathcal{B}, B^{e}\right)+\mathrm{Q}\left(\mathcal{B}, B^{e}\right)
$$

where:

$$
\mathrm{P}\left(\mathcal{B}, B^{e}\right)=\int_{\mathcal{D}} \rho\left[\left\langle\mathbf{f}_{B}^{m}, \mathbf{v}\right\rangle+\left\langle\boldsymbol{\zeta}, \boldsymbol{\ell}_{\mathbf{c}}\right\rangle\right] d V+\int_{\partial \mathcal{D}}\left[\langle\mathbf{t}, \mathbf{v}\rangle+\left\langle\boldsymbol{\zeta}, \mathbf{m}_{\mathbf{c}}\right\rangle\right] d A
$$

if $\boldsymbol{\zeta}(\mathbf{x}, t)$ is the vorticity vector field, while $\mathbf{m}_{\mathbf{c}}$, and $\boldsymbol{\ell}_{c}$ are the surface couples and body moments respectively. $\mathbf{f}_{B}^{m}$ denotes the body force per unit mass. The local stress vector is denoted $\mathbf{t}(\mathbf{x}, t)$ and relates to the stress tensor, $\mathbf{T}(\mathbf{x}, t)$, via the theory of Cauchy. Also

$$
\mathrm{Q}\left(\mathcal{B}, B^{e}\right)=-\int_{\partial \mathcal{D}}\langle\mathbf{q}, \mathbf{n}\rangle \mathbf{d}
$$

since heat sources and radiant heat transfer is not considered herein. $\mathbf{q}(\mathbf{x}, t)$ denotes the local heat flux vector and vanishes on the boundary $\partial 00$. With the 
same definition as in equation $(4), \mathrm{A}(00 ; t)$, holds for the universal body, 0 , if an integral over $O$ is defined as:

$$
\int_{O_{0}}(\cdot) d m \equiv \sum_{i}\left[\int_{\mathcal{B}_{\mathrm{i}}}(\cdot) d m\right]=\int_{\mathcal{B}}(\cdot) d m+\sum_{i}^{\prime} \int_{\mathcal{B}_{\mathrm{i}}}(\cdot) d m
$$

where the summation, $\Sigma$, is taken over all bodies $\mathcal{B}_{i}$ in the universe and the summation $\Sigma^{\prime}$ over all bodies external to $\mathcal{B}$. The void, $\mathcal{O}$, has no mass and so is not included in these integrals over the universe.

Following Clausius, introduce the basic requirement:

Axiom 2.1 An identity

The equality:

$$
\mathrm{A}(00 ; t) \equiv 0
$$

acts as constraint on the universal body 00 .

In general, the quantity $\mathrm{A}(\mathcal{B} ; t)$ is not identically zero due to the transfer of thermal and mechanical energy between the body $\mathcal{B}$ and its exterior $B^{e}$. However, it is assumed that there are no agencies exterior to 00 that may influence the universe either as energy transfer or via the action of forces and torques. Hence the function $\mathrm{A}(00 ; t)$ is time invariant and can be written simply as $\mathcal{A}(00)$.

First, consider the transformation of $\mathrm{A}(0)$ under the axial group.

$$
\mathrm{A}(00) \mapsto \mathrm{A}(00)+I_{a}(00)
$$

where the integral $I_{a}(00)$ has the form:

$$
I_{a}(00)=\int 00\langle\mathbf{Z} \mathbf{x}, \mathbf{a}+\mathbf{Z} \mathbf{v}\rangle \mathbf{d m} \equiv \mathbf{0}=\int 00\langle\mathbf{r}, \mathbf{a} \times \mathbf{x}\rangle \mathbf{d m}
$$

from equation (3), axiom 2.1 and the geometry of the vectors involved. Next, consider the invariance of $\mathrm{A}(0)$ under the Galilean group when equation (3) shows that:

$$
\mathrm{A}(00) \mapsto \mathrm{A}(00)+I_{g}(00)
$$

with $I_{g}$ given as:

$$
I_{g}(00)=\int 00\left\langle\mathbf{a}, \mathbf{Q}^{T} \mathbf{V}_{T}\right\rangle d m
$$

Axiom 2.1 requires that there be covariance for $\mathrm{A}(00)$. Hence, both $I_{a}(00)$ and $I_{g}(00)$ must vanish identically.

Consider the two constraints (8) and (10) separately:

a). $I_{a}(00)$ : The integral vanishes identically from the geometry of constant angular velocity rotation for a rigid universe on the subspace $\operatorname{span}\{\mathbf{r}\}^{\perp}$ through 
the mass center of 00 . That is, rotation of a rigid body with an arbitrary, constant, angular velocity is a privileged state of motion that satisfies axiom 2.1. This finding is not an explicit expression for angular motion, in the sense of equation (15) below, for linear motion.

b). $I_{g}(0)$ : This condition, $I_{g}(0)=0$, holds iff $\int_{00}\left\langle\mathbf{a}, \mathbf{Q}^{T} \mathbf{V}_{T}\right\rangle d m \equiv 0$ from equation (10). Since the vector $\mathbf{V}_{\mathbf{T}}$ is an arbitrary constant and the rotation $\mathbf{Q}$ is independent of location in 0 , there is: $\left\langle\mathbf{Q}^{T} \mathbf{V}_{T}, \mathbf{g}(0)\right\rangle=0$. This result has used the definition in equation (11), below, as applied to the entire universe 00 .

There is, as introduced in Moulden [9] and [10], the definition of an ancillary vector of some significance:

Definition 2.3 An ancillary vector $\mathrm{g}(\mathcal{B})$

Introduce the vector valued kinematic quantity, $\mathbf{g}(\mathcal{B})$, for any $\mathcal{B} \prec 0$, in the inertial frame $\Lambda_{c}$ :

$$
\mathbf{g}(\mathcal{B})=-\frac{d}{d t} \int_{\mathcal{B}} \mathbf{v}(\mathbf{x}, t) d m=-\frac{d}{d t} \mathbf{M}_{o m}(\mathcal{B}) \equiv-\int_{\mathcal{B}} \mathbf{a}(\mathbf{x}, t) d m
$$

so that:

$$
\mathbf{g}(\mathcal{B})=\mathbf{f}_{I}\left(\mathcal{B}, \mathcal{B}^{e}\right)
$$

from Lemma 1.1 since $M(\mathcal{B})$ is an invariant of the motion.

Physical significance will be attached to the vector $\mathrm{g}(\mathcal{B})$ in what follows. In fact it will play a central role in the discussion of the linear Mach's Principle. It is assumed in the above that $\mathbf{v}(\mathbf{x}, t)$ is a smooth function of space and time so that the acceleration field, $\mathbf{a}(\mathbf{x}, t)$, is well defined. This regularity assumption, along with uniqueness of the field equations, must be checked for consistency a posteriori but that aspect of the theory is not complete (particularly in fluid mechanics; see Lions [6], for example). It is concluded, from equation (10), that for Galilean covariance:

Result 2.1 Vanishing of the vector $\mathbf{g}(0)$.

$$
I_{g}(0)=0 \quad \Rightarrow \quad \mathrm{g}(00) \equiv-\int_{0} \mathbf{a} d m=\mathbf{0}
$$

and $d\left[\mathbf{M}_{o m}(00)\right] / d t=\mathbf{0}$ from equation (11).

Hence the momentum of the entire universe, $\mathbf{M}_{o m}(0)$, is time invariant as a constraint on possible motions of the universe and is consistent with the classical result for a system of point masses. This is, of course, also consistent with there being no external forces acting upon $O D$ as required in definition 1.1. 
Some invariance properties of the function $\mathrm{A}(00)=\mathrm{P}\left(00,00^{e}\right)+\mathrm{Q}\left(00,00^{e}\right)$ can be written down using the definitions in equation (3). Note that the surface integrals in equations (5) and (6) vanish on the boundary of 00 . O $0^{e}$ denotes the exterior of the universe 00 . Hence, $Q\left(00,00^{e}\right)$ vanishes identically while the quantity:

$$
\mathrm{P}\left(\infty, 00^{e}\right)=\int_{00} \rho\left[\left\langle\mathbf{f}_{B}^{m}, \mathbf{v}\right\rangle+\left\langle\boldsymbol{\zeta}, \boldsymbol{\ell}_{c}\right\rangle\right] d V
$$

only vanishes due to the equality $\mathrm{A}(00) \equiv 0=\mathrm{P}\left(00,00^{e}\right)$ that was specified in axiom 2.1 above.

\section{The Linear Principle of Mach}

Assuming that the body moments, $\boldsymbol{\ell}_{c}$, are frame indifferent under $\mathbb{G a}$, equation (13) transforms as:

$$
\mathrm{P}\left(00,00^{e}\right) \mapsto \mathrm{P}\left(00,00^{e}\right)+\int_{00}\left\langle\mathbf{f}_{B}^{m}, \mathbf{V}_{T}\right\rangle d m=0
$$

under that group. Hence, for covariance there is: $\int_{00}\left\langle\mathbf{f}_{B}^{m}, \mathbf{V}_{T}\right\rangle d m=0$, or:

$$
\int_{00} \mathbf{f}_{B}^{m} d m \equiv \mathbf{0}
$$

since $\mathbf{V}_{T}$ is arbitrary.

In the words of Bondi [3], the principle of Mach may be summarized as: "The local inertial frame is determined by some average of the motion of the distant astronomical objects". This can be shown from equation (12) where the statement $\mathbf{g}(0) \equiv \mathbf{0}$ followed from Galilean covariance of the action $\mathrm{A}(0)$. Hence the Newtonian statement:

Lemma 3.1 Since $\mathrm{A}(0)$ is Galilean invariant the linear principle of Mach, in Theorem 1.1(b) holds true.

Proof. There is from the linearity of the vector $\mathrm{g}(\mathcal{B})$ in definition 2.3 and using result $2.1\left(\right.$ with $\mathbf{f}_{I}\left(\mathcal{B}, B^{e}\right)$ the inertial force of body $\left.\mathcal{B}\right)$ :

$$
\mathbf{g}(0) \equiv \mathbf{0}=\mathbf{g}(\mathcal{B})+\mathbf{g}\left(B^{e}\right) \Rightarrow \mathbf{f}_{I}\left(\mathcal{B}, B^{e}\right)-\frac{d}{d t} \int_{\mathcal{B}^{e}} \mathbf{v} d m \equiv \mathbf{0}
$$

as linear momentum is additive over 0 .

Lemma 3.1 provides the continuum thermo-mechanics statement of the linear Mach's principle for Newtonian mechanics in the model universe considered herein. 
Result 3.1 The integral in equation (15) is invariant under Galilean transformations (since, by proposition 2.1, $\mathbf{g}(\mathcal{B})$ is frame indifferent under $\mathbb{G}$ a for any $\mathcal{B})$ but not under more general space-time transformations.

this result responds to Theorem 1.1(b), above, and goes some way towards resolving the concerns of Mach. Equation (15) is just a representation of the inertial force but is, in no way, an explanation for the existence of inertia: this form of the principle of Mach does not explain inertial effects which are distinct from gravitational attraction.

More can be said about Theorem 1.1(b). Equation (15) shows directly that the inertial force vanishes if the integral, $I_{u}$, given as:

$$
I_{u}=\int_{\mathcal{B}} \mathbf{v} d m \Rightarrow \mathbf{f}_{I}\left(\mathcal{B}, B^{e}\right)=-\frac{d}{d t} I_{u}
$$

is a constant. In that case the given body $\mathcal{B}$ would continue to move with whatever initial velocity, $\mathbf{v}_{0}$, it may possess.

The inertial force vector, $\mathbf{f}_{I}\left(\mathcal{B}, B^{e}\right)$, depends only on the rate of change of linear momentum of the exterior body $B^{e}$. It can be noted that Noll [12] made a similar observation from a different perspective. The result in lemma 3.1 can also be construed as a special case of the third axiom of Newton. It is important to note here that the second axiom of Newtonian mechanics has not been required: the forces arising in that axiom are not part of the present discussion of linear inertia. Indeed, the present result depends only upon the first axiom of Newtonian mechanics (the existence of the groups $\mathbb{G a}$ and $\mathbb{G z}$ ) and energy conservation via the $\mathrm{A}(\mathcal{B})$ function of definition 2.2 .

\section{The Angular Principle of Mach}

Newton did, as recorded above, in the Principia, introduce the notion of rotational inertia. This comment of Newton has received very little recognition but does point to an angular inertial effect that needs discussion in the spirit of Mach (in addition to the result of theorem 1.1(c) that was noted above).

Angular motion, in the present context, appears to have a different character within the field equations than does linear motion. Under the group $\mathbb{G} z$, equation (13) requires the transformation:

$$
\mathrm{P}\left(\infty, 0^{e}\right) \mapsto \mathrm{P}\left(\infty 0,00^{e}\right)+\int_{00}\left[\boldsymbol{\ell}_{c}+\mathbf{x} \times \mathbf{f}_{B}^{m}\right] d m=\mathbf{0}
$$

using arguments similar to those for equation (14) above, there is:

$$
\int_{00}\left[\boldsymbol{\ell}_{c}+\mathbf{x} \times \mathbf{f}_{B}^{m}\right] d m=\mathbf{0}=\int_{00}[\mathbf{a} \times \mathbf{x}] d m
$$


where the last equality follows from the angular momentum equality for the entire universe. Once the given body $\mathcal{B} \prec O$ is set in rotation, at constant angular velocity about a fixed axis through the mass center of that body, it will continue to rotate until some other torque is applied. Constraint (17) will hold during that motion. That is:

$$
\int_{\mathcal{B}}\left[\boldsymbol{\ell}_{c}+\mathbf{x} \times \mathbf{f}_{B}^{m}\right] d m=-\int_{\mathcal{B}}\left[\boldsymbol{\ell}_{c}+\mathbf{x} \times \mathbf{f}_{B}^{m}\right] d m
$$

and:

$$
\int_{\mathcal{B}}[\mathbf{a} \times \mathbf{x}]\left[d m=-\int_{\mathcal{B}} \mathbf{a} \times \mathbf{x}\right] d m
$$

to provide two statements of the Mach principle for angular motion.

\section{Final Remarks}

A Newtonian version of the principle of Mach has been established and adds insight into the structure of Newtonian mechanics on a global scale but does not explain body inertia.

It has been found that this Newtonian continuum mechanics version of the Mach principle depends upon assumptions made about the nature of the universe. These assumptions include:

i). The Clausius inductive statement of energy invariance for the universal body. The cosmological findings of dark matter and dark energy in the universe certainly call this into question in the classical form of axiom 2.1.

ii). The universe is assumed to be static. No agency exists outside of the universe,,$O$, to change the interior properties of that universe.

The above development is based upon Newtonian mechanics and neglects all electromagnetic and quantum effects. In this sense it is a poor model that omits very significant phenomenology from the real universe. Thus, while a Newtonian version of the principle of Mach can be constructed, it need not reflect the reality of the universe around us.

\section{References}

[1] Barbour, J. B. The Discovery of Dynamics, Oxford University Press, (2001).

[2] Berkeley, G. A treatise Concerning the Principles of Human Knowledge (1734), (Reissued by Oxford University Press, (Dancy, J. (Ed).) (1998). 
[3] Bondi, H. Cosmology, Cambridge University Press, (1960).

[4] Cohen, I. B. and Whitman, A. Isaac Newton: The Principia, University of California Press, (1999).

[5] Earman, J. World Enough and Space-Time, MIT Press, (1989).

[6] Lions, P-L. Mathematical Topics in Fluid Mechanics, vol. 1. Incompressible models, Oxford University Prss, (1996).

[7] Mach, E. The Science of Mechanics (First English Edition, 1919) (fourth German edition 1883), (1901). Reissued by Barnes and Noble, (2009).

[8] Mach, E. History and Root of the Principle of the conservation of Energy 2nd. Ed., Jourdain, P.E.B, (Trans) (1911).

[9] Moulden, T. H. On The first Axiom of Newtonian Continuum Mechanics, Int. J. Pure and Appl. Math. 77 No. 2, (2012), 199-212.

[10] Moulden, T. H. Mach's Principle in Newtonian Continuum Mechanics Proc. 9th HEFAT Conference, (2012).

[11] Newton, I. Philosophice Nateralis Principia Mathematica, Guil. and Jon. Innys, Regiæ Societatis Typographos, 3rd Edition, (1726).

[12] Noll, W. La méchanique classique, basée sur un axiome d'objectivité, Colloque International, Paris, 1959, (1963), 47-56. 
